\title{
Escolha do modelo de turbulência para um leito fluidizado
}

\author{
Choice of turbulence model for a fluidized bed \\ M. C. S. Sant'Anna ${ }^{1 *}$; S. M. Sarmento ${ }^{1}$; G. F. Silva ${ }^{2}$; R. A. Medronho ${ }^{3} ;$ S. \\ Lucena $^{1}$ \\ ${ }^{1}$ Departamento de Engenharia Química/Laboratório de Controle Avançado e Otimização de Processos/ \\ Universidade Federal de Pernambuco,50670-901, Recife-PE, Brasil \\ ${ }^{2}$ Departamento de Engenharia Química/Laboratório de Tecnologias Alternativa/ Universidade Federam de Sergipe, \\ 49200-000, São Cristóvão-SE, Brasil \\ ${ }^{3}$ Escola de Química/Laboratório de Fluidodinâmica Computacional/ Universidade Federal do Rio de Janeiro, \\ 21941-901, Rio de Janeiro-RJ, Brasil \\ *mikelecandida@gmail.com
}

\begin{abstract}
O leito fluidizado proporciona um excelente contato gás-sólido e elevadas taxas de transferência de calor e massa. A fim de otimizar os processos nas últimas décadas, intensificaram-se o uso de técnicas computacionais como a Fluidodinâmica Computacional, CFD, que resolve as equações de conservação através da discretização, no método dos volumes finitos. Nas simulações foram utilizadas o pacote comercial ANSYS FLUENT 15.0 e sistema de simulação contendo ar e areia. O teste de Modelo foi realizado para identificar o modelo de turbulência mais adequado para as simulações (k-દ ou SST). A geometria foi construída em $2 \mathrm{D}$ e foi realizado um teste de malha, para verificar se a malha influenciava nos resultados. A interação entre as fases foi determinada pela equação Syamlal-O'Brien e o valor do coeficiente de restituição partícula-partícula de 0,9 . A viscosidade das partículas foi calculada pela Teoria Cinética Granular. A velocidade de entrada do ar foi de $0,38 \mathrm{~ms}^{-1}$. Através da análise de expansão do leito e da queda de pressão optou-se por utilizar o Modelo k- $\varepsilon$ nas simulações seguintes, porque apresentou resultados concordantes com menor esforço computacional, em comparação com o modelo k- $\omega$. A malha três foi escolhida.
\end{abstract}

Palavras-chave: Fluidização, gaseificação, CFD, hidrodinâmica

The fluidized bed provide an excellent gas-solid contact and high rate of heat and mass transfer. In order to optimize the processes in recent decades intensified the use of computational techniques such as Computational Fluid Dynamics, CFD, that solves the equations of conservation through the discretization of the finite volume method. In the simulations of the computational system, package ANSYS FLUENT 15.0 and simulation system involving air and sand. Model test was performed to identify the most appropriate turbulence model to simulate (k-e or SST). The geometry 2D was constructed and conducted a mesh test, to see if the mesh influenced the results. The interaction between the phases was determined by the equation Syamlal-O'brien and the value of the coefficient of restitution particle-particle was equal to 0.9 . The viscosity of the particles was calculated by Kinetic Theory Granular. The inlet velocity of the air it was $0.38 \mathrm{~ms}^{-1}$. Through the analysis of charts bed expansion and pressure drop, coupled with analysis of the volume fraction and pressure profiles, it was decided to be used in the following simulations the model $\mathrm{k}-\varepsilon$ because it has concordant results with less computational effort, compared with the model $\mathrm{k}-\omega$. The mesh three was chosen.

Keywords: fluidization, gasifier, CFD, hydrodinamic

\section{INTRODUÇÃO}

Leitos fluidizados são utilizados para a geração de energia através da combustão e gaseificação de biomassa ou carvão em diferentes processos: indústrias químicas, farmacêuticas, alimentos, entre outras. A modelagem numérica de reatores de leito fluidizado é muito desafiadora, devido ao complexo comportamento do escoamento e das interações das partículas sólidas [1].

Nas últimas décadas, com o intuito de otimizar estes processos foi intensificado uso de técnicas computacionais como CFD que resolve as equações de conservação através do método de discretização dos volumes finitos. $\mathrm{O}$ aumento na capacidade de cálculo possibilitou a modelagem numérica em escoamentos complexos como leitos fluidizados. 
Duas diferentes abordagens podem ser utilizadas para a modelagem de escoamentos multifásicos (neste trabalho o escoamento gás-sólido) em CFD: modelo Lagrangeano (método discreto baseado na mecânica do continuo) [2] e o modelo Euleriano (baseado na hipótese de que as partículas são tratadas como um continuo e que as partículas apresentam propriedades similares as propriedades dos fluidos) [3].

Após definir o tipo de abordagem para o escoamento multifásico (abordagem Euleriana) e definir a importância da utilização da Teoria Cinética Granular, é necessário escolher o modelo de turbulência que será utilizado no problema.

A escolha de um modelo ideal para o escoamento turbulento deve introduzir o mínimo em complexidade, enquanto adquire a essência física relevante. Por ser um fenômeno complexo, é importante se ter uma grande quantidade de informações, assim, espera-se que quanto mais complexo seja o problema a ser resolvido, mais sofisticado deverá ser o método de solução a ser adotado. Logo, o requisito de simplicidade do modelo de turbulência será relativo à complexidade do problema a ser solucionado [4]. O objetivo deste trabalho foi realizar o teste de modelo de turbulência para verificar qual modelo se adequa mais as simulações hidrodinâmicas de um leio fluidizado borbulhante, para isso realizou-se simulações para os modelos $k-\varepsilon$ e SST.

Após a definição do Modelo de turbulência, foi realizado um teste de malha. A malha é a principal responsável pela qualidade da solução, e deve-se atentar à sua construção, a fim de que ela não influencie no resultado, sendo, tão somente, um instrumento para atingir o mesmo. $\mathrm{O}$ leito fluidizado foi subdividido em pequenos elementos onde foram empregados métodos numéricos para a solução das equações de transporte. A precisão da solução melhora com o refino da malha, como consequência deste refino o número de elementos aumenta e o esforço computacional também. Com isso, deve-se encontrar uma malha que garanta a qualidade da resposta desejada com menor custo computacional.

\section{MATERIAL E MÉTODOS}

O software comercial utilizado nas simulações foi o ANSYS FLUENT 15.0 que utiliza o método dos volumes finitos para a simulação numérica. As simulações foram realizadas em um computador com configuração básica de processador de Intel (R) Xeon (R) CPU E31240 @ $3,30 \mathrm{GHz}$ e memória RAM de $16 \mathrm{~Gb}$.

O leito fluidizado simulado foi construído utilizando-se o software ANSYS CFX 15.0, sendo um pseudo 2D, com as seguintes dimensões: $1 \mathrm{~m}$ de altura e $0,58 \mathrm{~m}$ de largura e profundidade de $0,001 \mathrm{~m}$.

\subsection{CFD Modeling}

A simulação do leito fluidizado foi realizada resolvendo as equações da conservação de massa, momentum e energia através da utilização do software de CFD ANSYS Fluent 15.0.

A abordagem Euleriana foi adotada neste trabalho, juntamente com a Teoria Cinética Granular descrita em [5].

Equação da conservação de massa (o subscrito i pode ser para fase gás ou sólida):

$$
\frac{\partial}{\partial t}\left(\alpha_{i} \rho_{i}\right)+\nabla\left(\alpha_{i} \rho_{i} \vec{v}_{i}\right)=0
$$

Equação da conservação de momentum para fase gás (g) e fase sólida (s):

$$
\frac{\partial}{\partial t}\left(\alpha_{g} \rho_{g} \vec{v}_{g}\right)+\nabla\left(\alpha_{g} \rho_{g} \vec{v}_{g}^{2}\right)=-\alpha_{g} \nabla p+\nabla \bar{\tau}_{g}+\alpha_{g} \rho_{g} \vec{g}+K_{g s}\left(\vec{v}_{g}-\vec{v}_{s}\right)
$$




$$
\frac{\partial}{\partial t}\left(\alpha_{s} \rho_{s} \vec{v}_{s}\right)+\nabla\left(\alpha_{s} \rho_{s} \bar{v}_{s}^{2}\right)=-\alpha_{s} \nabla p-\nabla p_{s}+\nabla \overline{\bar{\tau}}_{s}+\alpha_{s} \rho_{s} \vec{g}+K_{g s}\left(\vec{v}_{g}-\vec{v}_{s}\right)
$$

As equações constitutivas foram necessárias para o fechamento das equações governantes. A viscosidade da partículas foi calculada pela Teoria Cinética Granular, as correlações foram descritas na Tabela 1.

Tabela 1: Equações de viscosidade

\begin{tabular}{ccc}
\hline Viscosidade & Modelo & Referência \\
\hline Viscosidade cisalhante & Syamlal-Obrien, 1989 & {$[2,6]$} \\
Viscosidade Colisional & Gidaspow et al., 1994 & {$[3,6]$} \\
Viscosidade cinética & Syamlal et al., 1993 & {$[2,6]$} \\
Viscosidade cisalhante friccional & Schaeffer, 1987 & {$[3,6]$} \\
Viscosidade global & Lun et al., 1984 & {$[3,6]$} \\
\hline
\end{tabular}

O valor do coeficiente de restituição partícula-partícula foi igual a 0,9 como utilizado por [2, $3,6,7,8]$.

A areia utilizada no leito do gaseificador é do tipo quartzosa, este material é necessário para melhorar a estabilidade térmica e dinâmica do sistema. O principal objetivo em alimentar a areia nestes equipamentos é promover a uniformidade térmica no circuito, evitando zonas frias que possam dificultar a ignição. Também insere-se maior massa deste material frente ao combustível, com o intuito de promover o correto balanço de pressão no sistema e fluxo de partículas [9]. A Tabela 2 sumariza as propriedades dos materiais aplicados nas simulações do leito fluidizado.

Tabela 2: Propriedades dos Materiais

\begin{tabular}{ccc}
\hline Properties & Value & Reference \\
\hline Densidade da areia $\left(\mathrm{kgm}^{-3}\right)$ & $2,50 \times 10^{3}$ & {$[5]$} \\
Diâmetro da partícula de areia $(\mu \mathrm{m})$ & $2,75 \times 10^{2}$ & {$[5]$} \\
Densidade do gás $\left(\mathrm{kg} \mathrm{m}^{-3}\right)$ & $1,22 \times 10^{0}$ & {$[5]$} \\
Calor específico do gás $\left(\mathrm{JKg}^{-1}-\mathrm{K}^{-1}\right)$ & $1,00 \times 10^{3}$ & {$[5]$} \\
Condutividade Térmica do gás $\left(\mathrm{Wm}^{-1}-\mathrm{K}^{-1}\right)$ & $2,40 \times 10^{-2}$ & {$[5]$} \\
Viscosidade do gás $\left(\mathrm{kg}^{-1} \mathrm{~ms}^{-1}\right)$ & $7,89 \times 10^{-5}$ & {$[5]$} \\
\hline
\end{tabular}

A resolução do problema foi realizada de forma segregada utilizando o método Phase Coupled SIMPLE para solução do acoplamento pressão-velocidade. Foi utilizada a discretização espacial de segunda ordem para todas as equações, exceto para a fração volumétrica que foi discretizada usando o método QUICK. Para a discretização temporal foi utilizada a discretização de segunda ordem implícita.

Foram realizados testes para alcançar um valor de time-step de modo a obter o menor número de Courant. Convencionou-se o passo de tempo (time-step) fixo de 5.10 ${ }^{-5}$ segundos com 100.000 iterações $(5,00 \mathrm{~s}$ de simulação).

\subsection{Teste de Malha}

O teste de malha consiste primeiramente em elaborar uma malha grosseira com o pequeno número total de elementos. A partir desta malha, criam-se malhas mais refinadas, aumentando o número total de elementos e, consequentemente, diminuindo o tamanho desses elementos. $\mathrm{O}$ teste de malha foi realizado utilizando quatro níveis de refinamento crescente. A malha utilizada foi composta por elementos hexaédricos. Os dados estatísticos das malhas refinadas foram apresentados na Tabela 3. 
Tabela 3: Estatística das malhas da geometria B

\begin{tabular}{ccccc}
\hline Parâmetros & \multicolumn{4}{c}{ Malha } \\
\cline { 2 - 5 } & Malha 1 & Malha 2 & Malha 3 & Malha 4 \\
Maxface size & 0,006 & 0,005 & 0,004 & 0,003 \\
Número de nós & 65.464 & 93.834 & 146.292 & 259.184 \\
Número de elementos & 32.301 & 46.400 & 72.500 & 128.731 \\
Tetraedros: & 0 & 0 & 0 & 0 \\
Pirâmides: & 0 & 0 & 0 & 0 \\
Hexaedros: & 32.301 & 46.400 & 72.500 & 128.731 \\
Poliedros: & 0 & 0 & 0 & 0 \\
\hline
\end{tabular}

A qualidade da malha é importante para a precisão e estabilidade da solução numérica. Diferentes critérios podem ser utilizados para verificar a qualidade da malha, neste trabalho foram utilizados os seguintes parâmetros: Skewness; Element Quality e Orthogonal Quality. A Figura 1 ilustra as malhas 1, 2, 3 e 4 . As condições para simulação do teste de malha foram as apresentadas no item 2.1 .
a) Malha 1
b) Malha 2
c) Malha 3
d) Malha 4

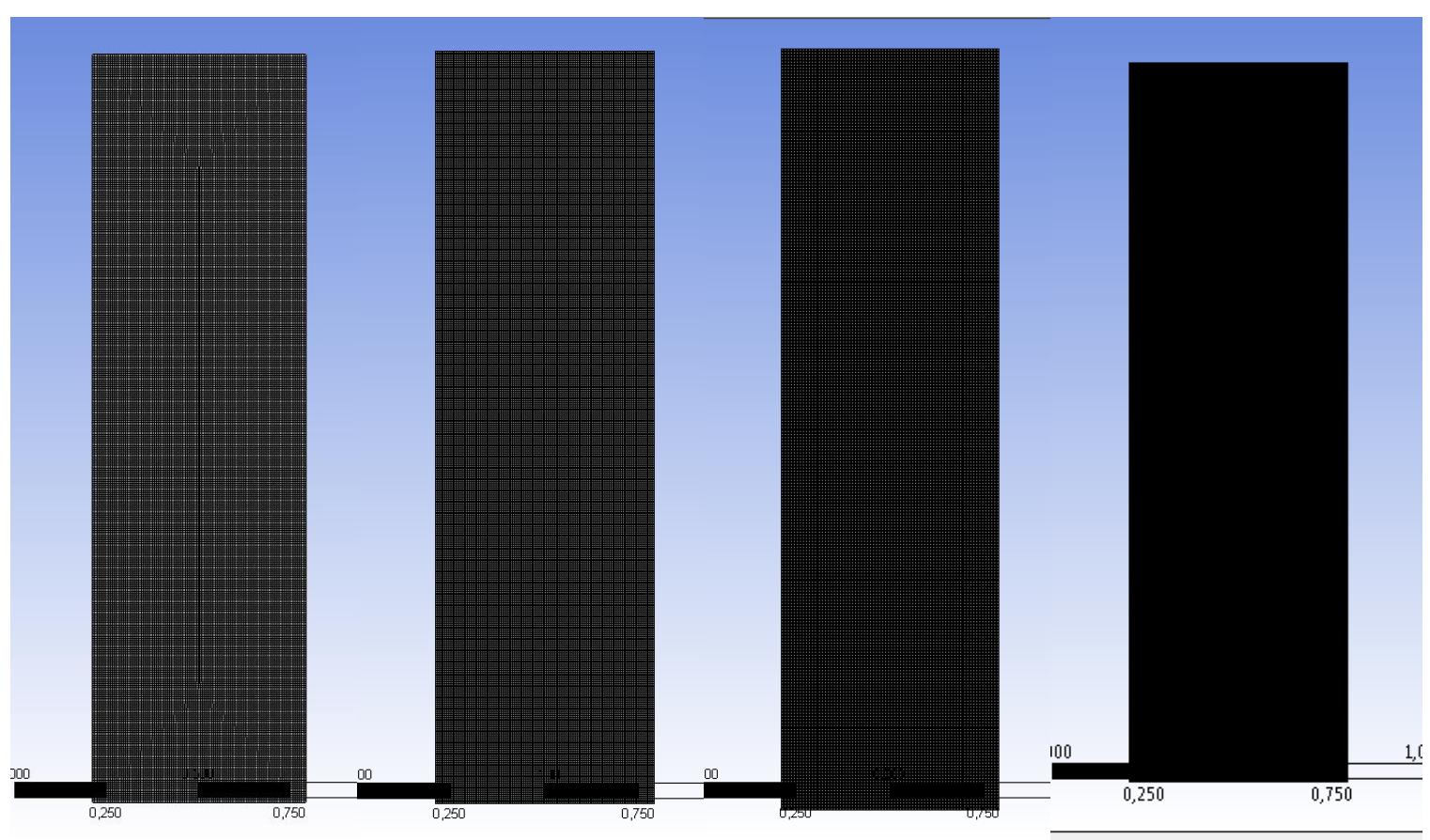

Figura 1: Malhas simuladas

\subsection{Teste de Modelo de Turbulência}

\subsubsection{Modelo $k-\varepsilon$}

O modelo $k$ - $\varepsilon$ apresenta vantagens por ser um modelo simples que necessita somente de condições iniciais e de contorno para sua aplicação. Possui uma excelente performance na maioria dos escoamentos relevantes na indústria, e, além disso, é o modelo mais validado com dados experimentais e industriais [10].

No modelo de turbulência $k-\varepsilon, k$ é a energia cinética de turbulência e é definida como a variação das flutuações de velocidade e $\varepsilon$ é a dissipação de energia turbulenta (a taxa na qual as flutuações de velocidade se dissipam). Este modelo é considerado robusto, econômico e razoavelmente preciso para uma larga faixa de escoamentos turbulentos. 
O modelo de turbulência $\kappa-\varepsilon$ Disperso utiliza para a fase contínua o modelo $\kappa-\varepsilon$ modificado com um termo adicional que inclui a transferência de momento turbulenta entre as fases. Para a fase dispersa, a turbulência é dada pela equação que se baseia na Teoria de Tchen de dispersão das partículas discreta em turbulência homogênea $[8,11]$.

A equação do transporte para $\kappa$ e para $\varepsilon$ disperso modificado para a fase contínua foram ilustradas nas Equações 27 e 28 [5].

$$
\begin{aligned}
& \frac{\partial}{\partial t}\left(\alpha_{g} \rho_{g} k_{g}\right)+\nabla \cdot\left(\alpha_{g} \rho_{g} \vec{U}_{g} k_{g}\right)=\nabla \cdot\left(\alpha_{g} \frac{\mu_{T, g}}{\sigma_{k}} \nabla k_{g}\right)+\alpha_{g} G_{k, g}-\alpha_{g} \rho_{g} \varepsilon_{g}+\alpha_{g} \rho_{g} \Pi_{k g} \\
& \frac{\partial}{\partial t}\left(\alpha_{g} \rho_{g} \varepsilon_{g}\right)+\nabla .\left(\alpha_{g} \rho_{g} \vec{U}_{g} \varepsilon_{g}\right)=\nabla \cdot\left(\alpha_{g} \frac{\mu_{T, g}}{\sigma_{\varepsilon}} \nabla \varepsilon_{g}\right)+\alpha_{g} \frac{\varepsilon_{g}}{k_{g}}\left(C_{1 \varepsilon} G_{k, g}-C_{2 \varepsilon} \rho_{g} \varepsilon_{g}\right)+\alpha_{g} \rho_{g} \Pi_{g g}
\end{aligned}
$$

As equações dos parâmetros utilizados no modelo de turbulência $\kappa-\varepsilon$ foram descritas na Tabela $4 \mathrm{e}$ as constantes do modelo foram descritos na Tabela 5.

\begin{tabular}{|c|c|c|}
\hline & ções & \\
\hline Viscosidade Turbulenta & $\mu_{T}=\rho C_{\mu} \frac{k^{2}}{\varepsilon}$ & $(06)$ \\
\hline $\begin{array}{l}\text { Produção da energia cinética } \\
\text { turbulenta na fase gasosa }\end{array}$ & $G_{k, g}=-\overline{\rho u_{i}^{\prime} u_{j}^{\prime}} \frac{\partial u_{i}}{\partial x_{i}}$ & $(07)$ \\
\hline $\begin{array}{l}\text { Influência da fase particulada na } \\
\text { fase gasosa }\end{array}$ & $\Pi_{k g}=\frac{\beta}{\alpha_{g} \rho_{g}}\left(k_{s g}-2 k_{g}+\vec{u}_{s g} \cdot \vec{u}_{d r}\right)$ & $(08)$ \\
\hline $\begin{array}{l}\text { Covariância entre as velocidades } \\
\text { das fases gasosa e particulada }\end{array}$ & $k_{s g}=2 k_{g}\left(\frac{b+\eta_{s g}}{1+\eta_{s g}}\right)$ & $(09)$ \\
\hline Velocidade de arraste & $\vec{u}_{d r}=-\left(\frac{D_{s}}{\sigma_{s g} \alpha_{s}} \nabla \alpha_{s}-\frac{D_{g}}{\sigma_{s g} \alpha_{g}} \nabla \alpha_{g}\right)$ & (10) \\
\hline Razão de tempo característico & $\eta_{s g}=\frac{\tau_{t, s g}}{\tau_{F, s g}}$ & (11) \\
\hline $\begin{array}{l}\text { Tempo de relaxamento das } \\
\text { partículas }\end{array}$ & $\tau_{F, s g}=\frac{\alpha_{g} \rho_{g}}{\beta}\left(\frac{\rho_{s}}{\rho_{g}}+C_{U}\right)$ & (12) \\
\hline Tempo integral Lagrangeano & $\tau_{t, s g}=\frac{\tau_{t, g}}{\sqrt{1+C_{\beta} \xi^{2}}} \quad \xi=\frac{\left|\vec{u}_{s g}\right| \tau_{t, g}}{L_{t, g}} C_{\beta}=1,8-1,35 \cos ^{2} \theta$ & (13) \\
\hline $\begin{array}{l}\text { Tempo característico da fase } \\
\text { contínua }\end{array}$ & $\tau_{t, g}=\frac{3}{2} C_{\mu} \frac{k_{g}}{\varepsilon_{g}}$ & (14) \\
\hline $\begin{array}{l}\text { Comprimento de escala dos } \\
\text { turbilhões da fase contínua }\end{array}$ & $L_{t, g}=\sqrt{\frac{3}{2}} C_{\mu} \frac{k_{g}^{\frac{3}{2}}}{\varepsilon_{g}}$ & (15) \\
\hline Difusividade de cada fase & $D_{s}=D_{t, s g}+\left(\frac{2}{3} k_{s}-b \frac{1}{3} k_{s g}\right) \tau_{F, s g} D_{t, s g}=\frac{1}{3} k_{s g} \tau_{t, s g}$ & (16) \\
\hline Turbulência para a fase dispersa & $k_{s}=k_{g}\left(\frac{b^{2}+\eta_{s g}}{1+\eta_{s g}}\right) b=\left(1+C_{U}\right)\left(\frac{\rho_{s}}{\rho_{g}}+C_{U}\right)^{-1}$ & (17) \\
\hline
\end{tabular}

Tabela 4: Equações do Modelo de Turbulência

Tabela 5: Constantes do modelo $\kappa-\varepsilon$

\begin{tabular}{ccccccccc}
\hline Constante & $C_{\mu}$ & $C_{1 \varepsilon}$ & $C_{2 \varepsilon}$ & $C_{3 \varepsilon}$ & $\sigma_{k}$ & $\sigma_{\varepsilon}$ & $\sigma_{s g}$ & $C_{U}$ \\
\hline Valores & 0,09 & 1,44 & 1,92 & 1,20 & 1,00 & 1,00 & 0,75 & 0,50 \\
\hline
\end{tabular}




\subsubsection{Modelo SST}

O modelo de turbulência SST de duas equações foi desenvolvido por Menter em 1994 no trabalho Two-Equation Eddy-Viscosity Turbulence Models for Engineerig Applications. A formulação do modelo SST foi baseada em experiências físicas e para prever soluções para os problemas típicos de engenharia. Ao longo das duas últimas décadas, o modelo foi alterado para descrever com mais precisão determinadas condições de fluxo. As duas variáveis calculadas são geralmente interpretadas como: $\mathrm{k}$ é a energia cinética turbulenta e $\omega$ é a taxa de dissipação de energia.

O SST é um modelo de turbulência amplamente utilizado e robusto de duas equações utilizado em CFD. O modelo combina o modelo de turbulência $\mathrm{k}-\varepsilon$ e o k- $\omega$ (que é usado na região interna da camada limite).

São duas equações de transporte para o modelo SST, sendo uma a equação da energia cinética turbulenta $(k)$, Equação 18 , e outra para a taxa de dissipação específica de energia cinética turbulenta $(\omega)$, Equação 19.

$$
\begin{aligned}
& \frac{\partial}{\partial t}(\rho k)+\frac{\partial}{\partial x_{j}}\left(\rho u_{j} k\right)=\frac{\partial}{\partial x_{j}}\left[\left(\mu+\frac{\mu_{t}}{\sigma_{k}}\right) \frac{\partial k}{\partial x_{j}}\right]+P_{k}-\beta^{\prime} \rho \omega k+P_{k b} \\
& \frac{\partial}{\partial t}(\rho \omega)+\frac{\partial}{\partial x_{j}}\left(\rho u_{j} \omega\right)=\frac{\partial}{\partial x_{j}}\left[\left(\mu+\frac{\mu_{t}}{\sigma_{\omega}}\right) \frac{\partial \omega}{\partial x_{j}}\right]+a \frac{\omega}{k} P_{k}-\beta \rho \omega^{2}+P_{\omega b}
\end{aligned}
$$

As equações dos parâmetros utilizados no modelo de turbulência SST foram descritas na Tabela 6 e as constantes do modelo foram descritas na Tabela 7.

\begin{tabular}{|c|c|c|}
\hline \multicolumn{3}{|c|}{ Equações } \\
\hline Term $\tilde{p}_{k}$ & $\tilde{p}_{k}=\min \left(P_{k}, 10 \varepsilon\right)$, onde $\varepsilon=\omega * \beta * k \quad \tilde{p}_{k}=\mu_{t} \frac{\partial u_{i}}{\partial x_{j}}\left(\frac{\partial u_{i}}{\partial x_{j}}+\frac{\partial u_{i}}{\partial x_{j}}\right)$ & (20) \\
\hline$v_{t}$ & $v_{t}=\frac{a_{1} k}{\max \left(a_{1} \omega, S F_{2}\right)} \quad v_{t}=\mu_{t} / \rho$ & (21) \\
\hline Termo $F_{1}$ & 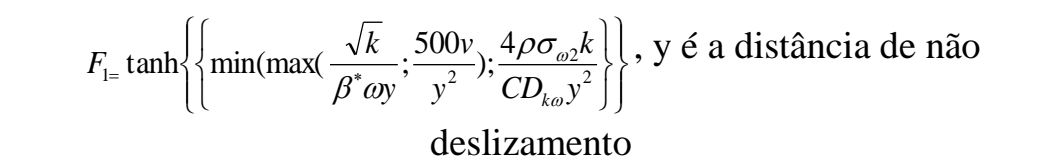 & (22) \\
\hline$C D_{k \omega}$ & $C D_{k \omega}=\max \left(2 \rho \sigma_{w 2} \frac{1}{\omega} \frac{\partial k}{\partial x_{i}} \frac{\partial \omega}{\partial x_{i}}, 10^{-10}\right)$ & (23) \\
\hline $\begin{array}{l}\text { Turbulence } \\
\text { viscosity }\end{array}$ & $\mu_{t}=\rho \frac{\alpha_{1} k}{\max \left(\alpha_{1} \omega\left(S_{0} S_{0}\right)^{1 / 2} F_{2}\right)},\left(S_{0} S_{0}\right)^{1 / 2}$ é uma medida invariante do tensores & (24) \\
\hline $\begin{array}{c}\mu_{t} \\
\text { Term } F_{2}\end{array}$ &  & (25) \\
\hline
\end{tabular}

Tabela 6: Parâmetros do modelo de turbulência

Table 7: The constant SST turbulence model

\begin{tabular}{cccccccccc}
\hline Constante & $\beta$ & $\alpha_{1}$ & $\beta_{1}$ & $\sigma_{k 1}$ & $\sigma_{\theta 1}$ & $\alpha_{2}$ & $\beta_{2}$ & $\sigma_{k 2}$ & $\sigma_{\theta 2}$ \\
\hline Valores & 0,09 & 0,55 & 0,07 & 0,85 & 0,50 & 0,44 & 0,08 & 1 & 0,86 \\
\hline
\end{tabular}




\section{RESULTADOS E DISCUSSÃO}

\subsection{Teste de Malha}

Os parâmetros Element Quality médio e Orthogonal Quality médio devem ser próximos a 1 e o Skewness médio próximo a 0 para que a malha tenha qualidade [5]. As malhas utilizadas no teste apresentaram valores próximos ao que foi recomendado (Tabela 8).

Tabela 8: Parâmetros que avaliam a qualidade das Malhas da geometria B

\begin{tabular}{lllll}
\hline Parâmetros & \multicolumn{4}{c}{ Malhas } \\
\cline { 2 - 5 } Skewness médio & Malha 8 & Malha 9 & Malha 10 & Malha 11 \\
Element Quality médio & $3,78 \mathrm{E}-04$ & $7,71 \mathrm{E}-07$ & $8,37 \mathrm{E}-07$ & $1,92 \mathrm{E}-04$ \\
Orthogonal Quality médio & 0,9986 & 0,9994 & 0,9994 & 0,9991 \\
\hline
\end{tabular}

Para observar a influência da malha nos resultados foram escolhidos os parâmetros de expansão do leito e queda de pressão. Foram construídas curvas de fração volumétrica de areia em função da altura do leito (Figura 2). Para confirmar a análise foram construídas curvas da variação de pressão ao longo da altura do leito para as diferentes malhas analisadas (Figura 3).

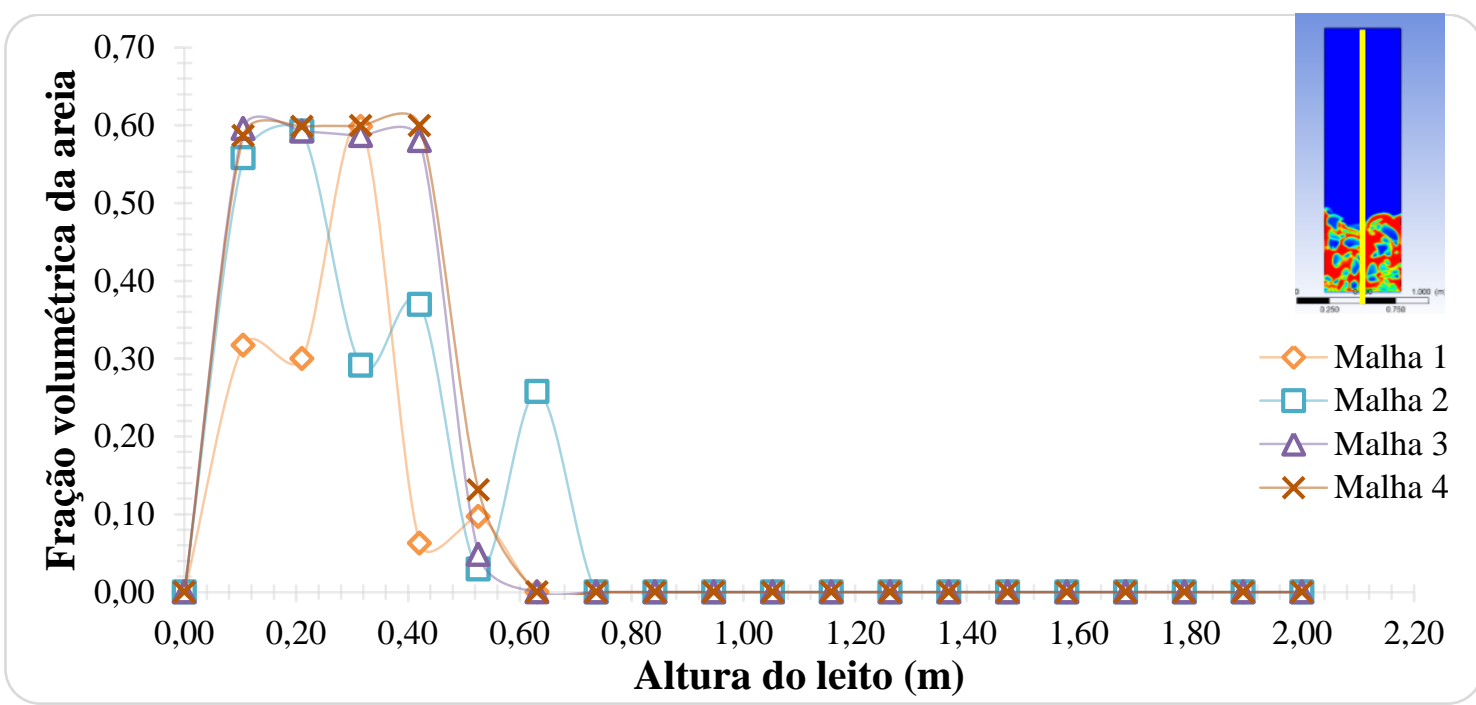

Figura 2: Expansão do leito para diferentes malhas $\left(v_{g}=0,38 \mathrm{~m} \cdot \mathrm{s}^{-1}\right)$.

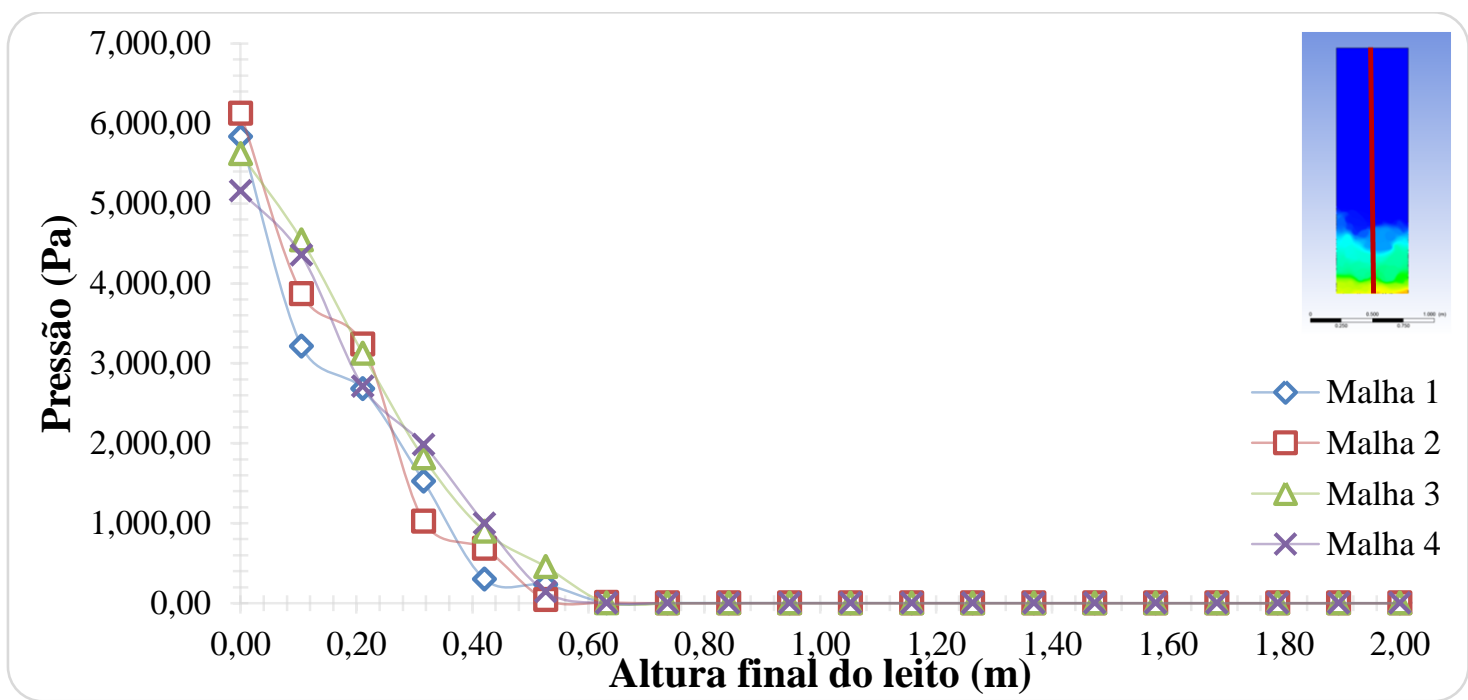

Figura 3: Pressão versus altura do leito para diferentes malhas $\left(v_{g}=0,38 \mathrm{~m} \cdot \mathrm{s}^{-1}\right)$. 
Pode-se observar nas Figuras 3 e 4 que para as diferentes malhas testadas as curvas das Malhas 3 e 4 foram bem próximas, quase coincidentes. Optou-se, então, pela Malha 3, em virtude desta apresentar um menor número de elementos e por consequência reduzir o esforço computacional.

\subsection{Teste de Modelo}

Para facilitar a comparação realizada para os dois modelos de turbulência foram construídas curvas de fração volumétrica de areia em função da altura do leito para os diferentes tempos de simulação $(0,12 ; 2,50$ e 10,00 s), usando os modelos de turbulência k-є e SST (Figura 4).

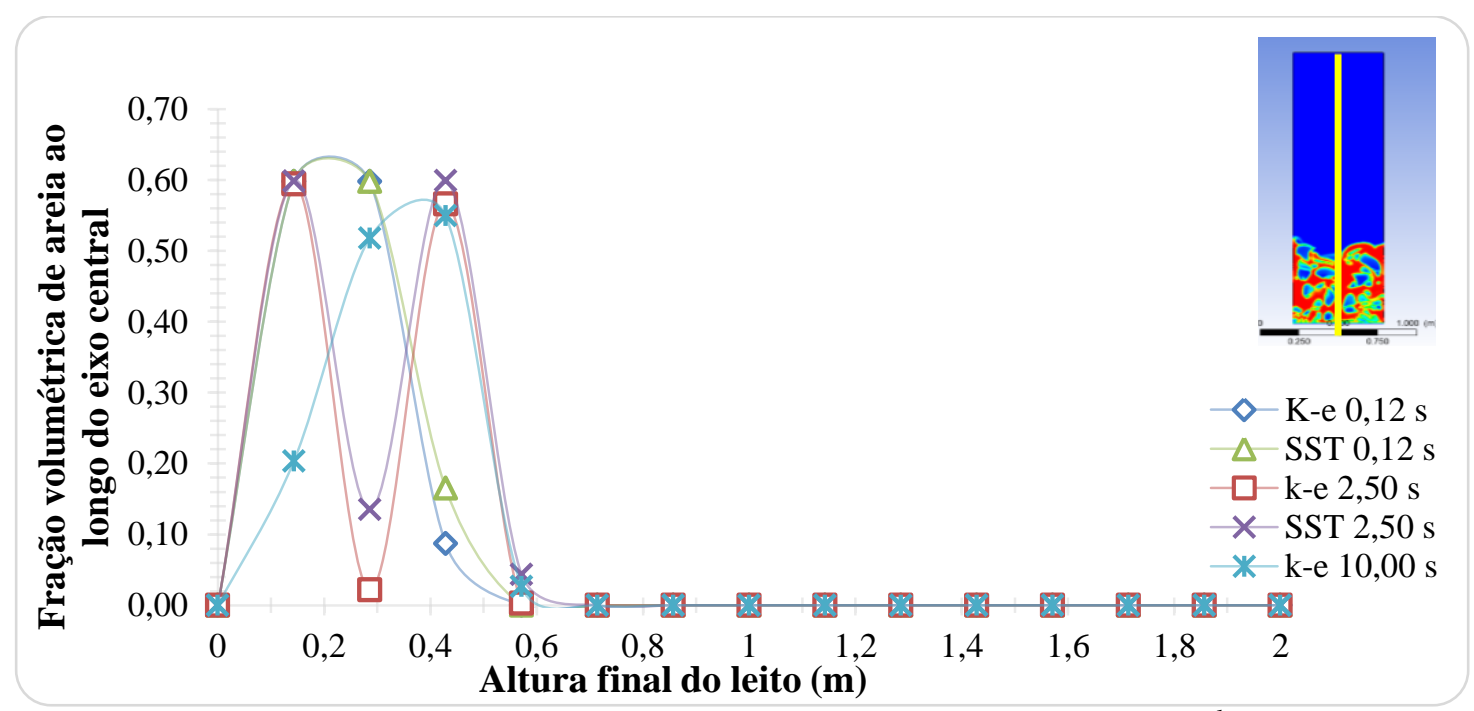

Figura 4: Expansão do leito para os modelos $k-\varepsilon$ e $k-\omega\left(v_{g}=0,38 \mathrm{~ms}^{-1}\right)$.

Na Figura 4 foi possível concluir que o emprego dos modelos k- $\varepsilon$ e SST levou a resultados similares para a porosidade do leito $(0,60)$. Aos $2,50 \mathrm{~s}$ de simulação, a fluidização já se encontrava em regime pseudo-estacionário e em regime borbulhante. Nesta situação, a expansão do leito não sofre mais diferenças significativas com o passar do tempo de simulação, o que foi comprovado com a simulação realizada para o modelo k- $\varepsilon$ para o tempo de 10,00 s. Concordando com os resultados observados por [12] que o padrão dinâmico regular foi estabelecido a partir de $2,00 \mathrm{~s}$ de simulação para um leito fluidizado borbulhante. A altura final do leito foi de $0,60 \mathrm{~m}$, o que representa uma expansão de $0,20 \mathrm{~m}$, uma vez que a altura inicial do leito fixo foi de $0,40 \mathrm{~m}$. A velocidade de entrada do gás utilizada nestas simulações foi de 0,38 $\mathrm{ms}^{-1}$.

Foi avaliado o comportamento da queda de pressão para os dois modelos, para facilitar a análise (Figura 5). A velocidade de entrada do gás utilizadas nestas simulações foi de $0,38 \mathrm{~ms}^{-1}$. 


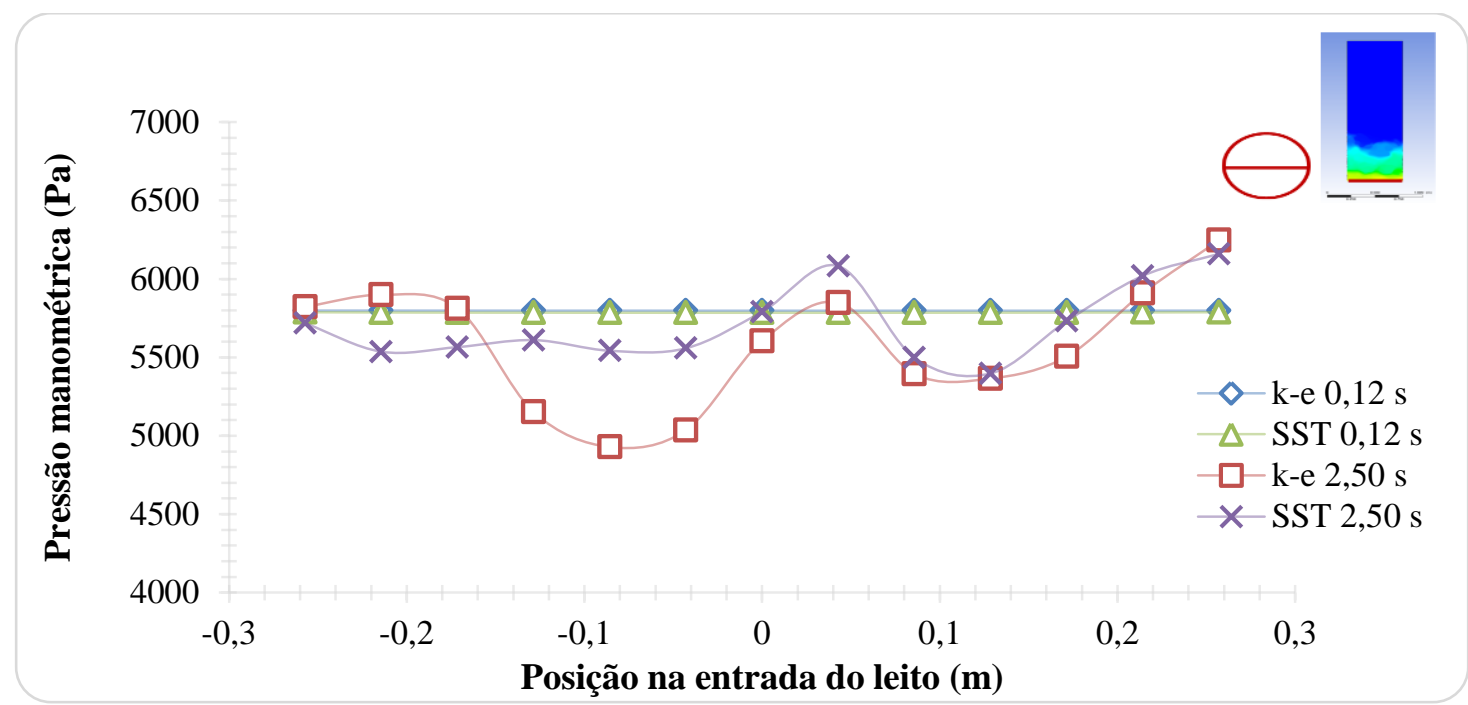

Figura 5: Pressão manométrica versus posição para os modelos de turbulência $k$-E e SST $\left(v_{g}=0,38 \mathrm{~ms}^{-1}\right)$.

As curvas foram construídas nos tempos de 1,25 e 2,50 s, ilustrando como os modelos se comportam no início da simulação (enquanto o leito ainda encontra-se em fixo), e quando o sistema alcança o regime borbulhante Os valores obtidos para a pressão na linha de entrada representam a própria queda de pressão do leito, já que na saída do sistema a pressão é zero.

O valor da pressão calculada através da equação de pressão foi de $5886 \mathrm{~Pa}$, o valor experimental encontrado por [2] para a queda de pressão do leito foi de $5.000 \mathrm{~Pa}$. As curvas dos modelos oscilam entre 5.000 a $6.000 \mathrm{~Pa}$ para o tempo de $2,50 \mathrm{~s}$, no tempo de $0,125 \mathrm{~s}$ as curvas se sobrepõem. A queda de pressão se altera durante o período de fludização devido a distribuição de sólidos que se altera em função da variação no volume e porosidade do leito.

A Figura 6 ilustra os perfis longitudinais de pressão obtidos para os dois modelos nos diferentes tempos de simulação $(0,12 ; 2,50 ; 5,00 ; 10,00 \mathrm{~s})$. A velocidade de entrada do ar adotada nesta simulação foi de $0,38 \mathrm{~m} \cdot \mathrm{s}^{-1}$.

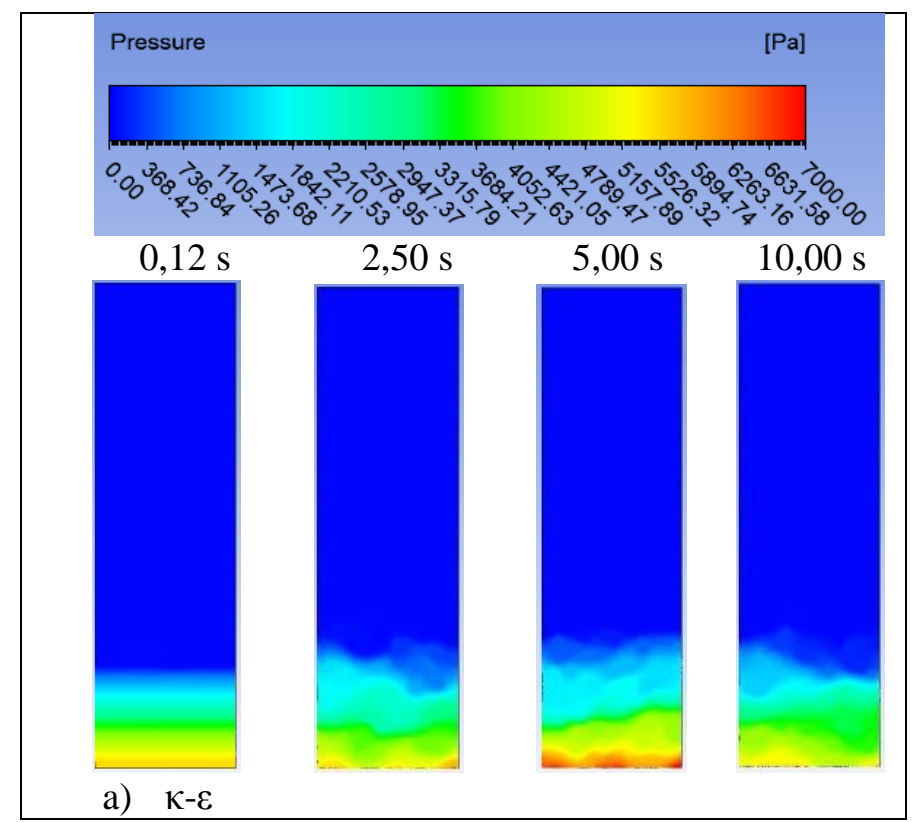




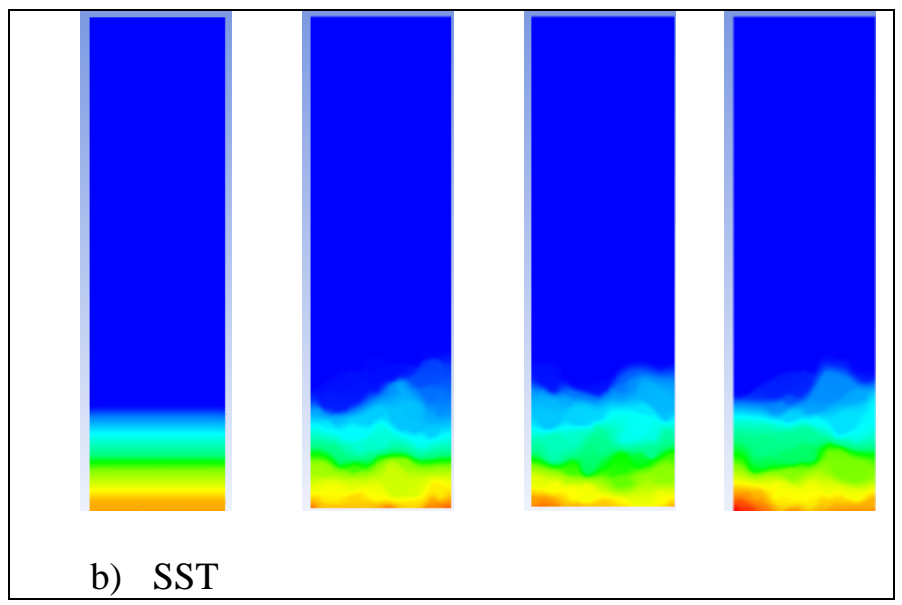

Figura 6: Perfis longitudinais de pressão para os modelos de turbulência: $a$ ) $\kappa-\varepsilon, b) S S T$

A Figura 7 ilustra os perfis longitudinais de fração volumétrica de areia obtidos para os dois modelos nos diferentes tempos de simulação $(0,12 ; 2,50 ; 5,00 ; 10,00 \mathrm{~s})$. A velocidade de entrada do ar adotada foi de $0,38 \mathrm{~m} \cdot \mathrm{s}^{-1}$.

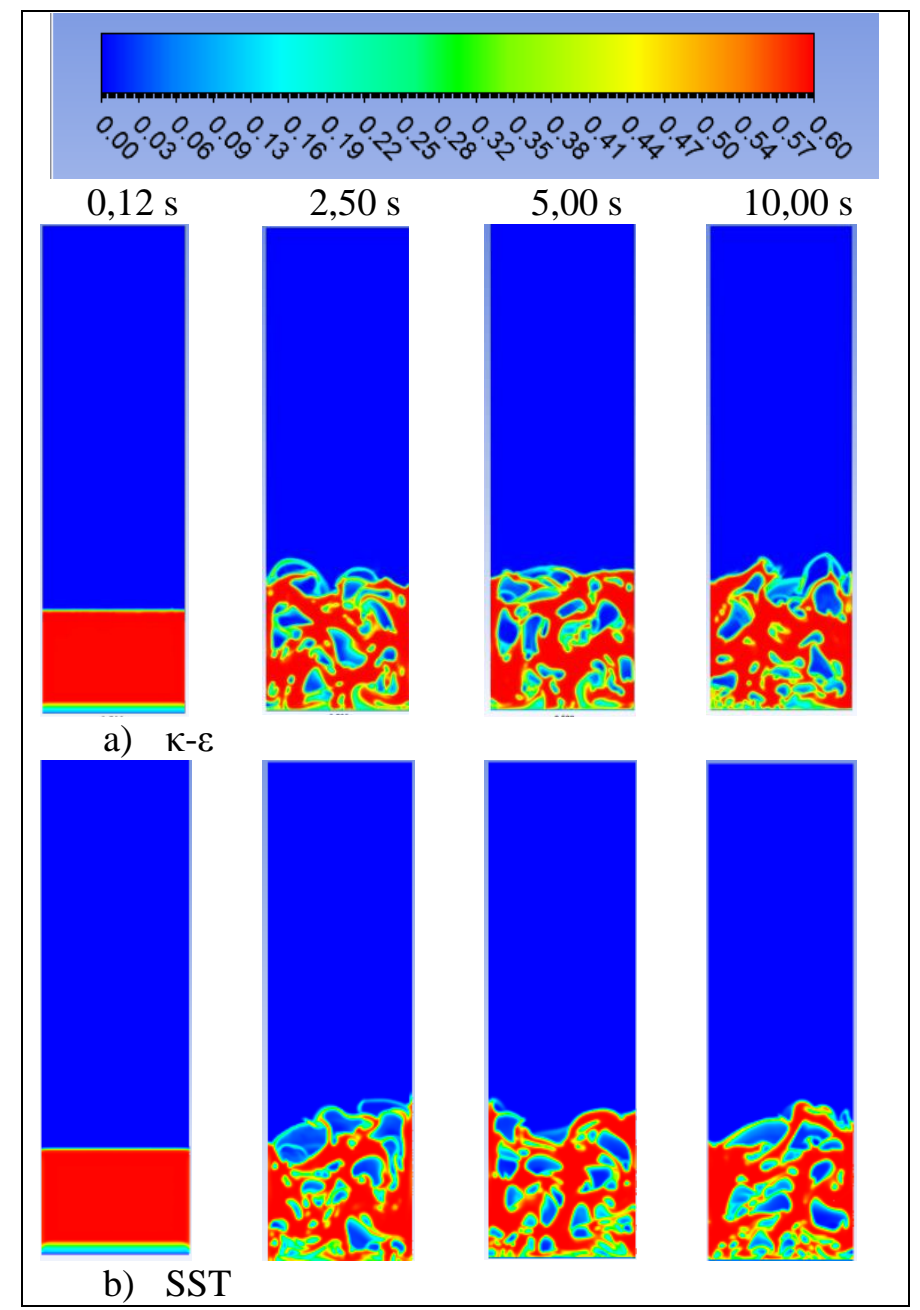

Figura 7: Perfis longitudinais de fração volumétrica de areia: a) $\kappa-\varepsilon, b)$ SST

Com a análise das curvas de expansão do leito e de queda de pressão, somado a análise dos perfis longitudinais de fração volumétrica e de pressão, optou-se pelo modelo k- $\varepsilon$, porque ambos os modelos apresentaram resultados similares para a expansão do leito, porém o modelo $\kappa-\varepsilon$ 
exige um menor esforço computacional, e a queda de pressão teve uma maior aproximação do valor experimental de Taghipour et al. (2005), quando comparado com o modelo SST.

\section{CONCLUSÃO}

Com a realização deste trabalho foi possível definir o modelo de turbulência adequado para as simulações seguintes que visam a otimização do sistema. Foi possível escolher uma malha que não altera os resultados e que demanda um menor esforço computacional.

Avaliar o comportamento da dinâmica do leito fluidizado é muito importante, pois reduz o esforço em experimentos. Com a utilização da técnica CFD é possível prever o comportamento dos sistemas, otimizar condições de fluidização.

Neste trabalho realizou-se o teste de modelo e de malha objetivando construir um setup que melhor representasse um gaseificador de leito fluidizado borbulhante. Esse trabalho serviu de subsidio para outros experimentos, que analisam as variáveis que possibilitam a otimização da operacionalização deste equipamento.

\section{REFERÊNCIAS BIBLIOGRÁFICAS}

1. Herzog N, Schreiber M, Egbers C, Krautz HJ. A comparative study of different CFD-codes for numerical simulation of gas-solid fluidized bed hydrodynamics. Computers and Chemical Engineering. 2012; 39:41-46, doi:10.1016/j.compchemeng.2011.12.002.

2. Taghipuor F, Ellis N, Wong C. Experimental and computational study of gas-solid fluidized bed hydrodynamics. Chemical Engineering Science. 2005 Jul; 60: 6857 -6867, doi:10.1016/j.ces.2005.05.044.

3. Adamczyk WP, Klimanek A, Bialecki RA, Wecel G, Kozolub P, Czakiert T. Comparison of the standard Euler-Euler and hybrid Euler-Lagrange approaches for modeling particle transport in a pilot-scale circulating fluidized bed. Particuology. 2013 Jun; 594: 1-9, doi:10.1016/j.partic.2013.06.008.

4. Lima Neto S C. Análise híbrida do escoamento turbulento em canais via modelos de turbulência de uma equação de transporte. [Tese]. João Pessoa (PB): Universidade Federal da Paraíba; 2007. 40-41p.

5. ANSYS Fluent 13.0. Theory Guide. Ansys Inc. USA. 2011.

6. Cornelissen JT, Taghipour F, Escudié R, Ellis N, Grace J R. CFD modelling of a liquid-solid fluidized bed. Chemical Engineering Science. 2007 Jul, 62: 6334-6348. doi:10.1016/j.ces.2007.07.014.

7. Pain CC, Mansoorzadeh S, Oliveira CRE. A study of bubbling and slugging fluidized beds using the two-fluid granular temperature model. International Journal of Multiphase Flow. 1984; 27: 527-551.

8. Faísca AT. A influência da geometria de saída na erosão em risers de fcc: um estudo com CFD. [Dissertação]. Rio de Janeiro (RJ): Universidade Federal do Rio de Janeiro; 2013. 14-35p.

9. Hodapp MJ. Simulação trifásica por técnicas de CFD da combustão de carvão mineral em leito fluidizado circulante. [Tese]. Campinas (SP): Universidade Estadual de Campinas; 2012. 5-25p.

10. Gomez DA. Análise do escoamento compressível de gás natural em espaços com restrições para elevação de petróleo. [Dissertação]. Rio de Janeiro (RJ): Universidade Federal do Rio de Janeiro; 2008. 35-39p.

11. Hinze JO. Turbulence. New York: McGraw-Hill Publishing Co; 1975.

12. Armstrong LM, Gu S, Luo KH. Study of wall-to-bed heat transfer in a bubbling fluidised bed using the kinetic theory of granular flow. International Journal of Heat and Mass Transfer. 2010 Jun; 53: 4949-4959. doi:10.1016/j.ijheatmasstransfer.2010.05.047. 